\title{
The Dietary Quality of Persons with Heart Failure in NHANES 1999-2006
}

\author{
Stephenie C. Lemon, $P h D^{7}$, Barbara Olendzki, $M P H, R D^{7}$, Robert Magner, BS ${ }^{7}$, Wenjun $L i, P h D^{7}$, \\ Annie L. Culver, BPharm², Ira Ockene, MD', and Robert J. Goldberg, PhD ${ }^{3}$ \\ 'Division of Preventive and Behavioral Medicine, S7-745, University of Massachusetts Medical School, Worcester, MA, USA; ${ }^{2}$ Mayo Clinic, \\ Rochester Methodist Hospital, Rochester, MN, USA; ${ }^{3}$ Division of Cardiovascular Medicine University of Massachusetts Medical School, \\ Worcester, MA, USA.
}

BACKGROUND: Dietary quality may impact heart failure outcomes. However, the current status of the dietary quality of persons with heart failure has not been previously reported.

OBJECTIVE: To describe sodium intake, patient factors associated with sodium intake and overall dietary quality in a national sample of persons with heart failure.

DESIGN: Analysis of repeated cross-sectional probability sample surveys using data from National Health and Nutrition Examination Surveys (NHANES) of 1999-2000, 2001-2002, 2003-2004 and 2005-2006.

PARTICIPANTS: The study sample consisted of 574 persons with self-reported heart failure (mean age= 70 years; $52 \%$ women).

MEASUREMENTS: Diet of each survey participant was assessed using single 24 hour recall. Dietary nutrients of interest included sodium, the mainstay of heart failure dietary recommendations, and additionally potassium, calcium, magnesium, fish oils, saturated fat and fiber. Specific dietary goals were based on established guidelines.

RESULTS: Mean sodium intake was 2,719 mg, with $34 \%$ consuming less than $2,000 \mathrm{mg}$ per day. Patient factors associated with greater sodium intake included male gender, lower education, lower income and no reported diagnosis of hypertension. Mean potassium intake was $2,367 \mathrm{mg} /$ day, with no differences by type of diuretic used or renal disease status. Adherence rates to established guidelines for other nutrients were $13 \%$ for calcium, $10 \%$ for magnesium, $2 \%$ for fish oils, $13 \%$ for saturated fat and $4 \%$ for fiber.

CONCLUSIONS: Dietary quality of persons with selfreported heart failure was poor. Public health approaches and clinical dietary interventions are needed for persons with this increasingly prevalent clinical syndrome.

KEY WORDS: dietary quality; heart failure; clinical dietary interventions; sodium intake.

Received November 12, 2008

Revised July 29, 2009

Accepted September 18, 2009

Published online October 31, 2009
$\mathrm{J}$ Gen Intern Med 25(2):135-40

DOI: $10.1007 / \mathrm{s} 11606-009-1139-\mathrm{x}$

( ) Society of General Internal Medicine 2009

\section{INTRODUCTION}

Heart failure is associated with considerable morbidity and mortality in the U.S. ${ }^{1}$ There is a growing body of evidence attesting to the importance of dietary factors in heart failure progression and outcomes. ${ }^{2-8}$ The American College of Cardiology/American Heart Association (ACC/AHA) joint guidelines recommend restricted sodium intake for patients with symptomatic, non-end-stage heart failure. ${ }^{9}$ These guidelines also endorse adherence to dietary guidelines for common underlying and comorbid conditions in chronic heart failure, including coronary heart disease, hypertension, hypercholesterolemia, and diabetes. The results of several recent studies have suggested that nutrients consistent with Dietary Approaches to Stop Hypertension (DASH) eating plan influence heart failure progression and associated outcomes. ${ }^{2-8}$ Additionally, omega-3 fish oil intake has been associated with a beneficial effect on heart failure outcomes. ${ }^{10}$

Despite the influence of dietary factors in determining disease outcomes in patients with heart failure, there is little understanding of the current dietary quality of persons with heart failure. ${ }^{11}$ The primary purpose of this analysis is to describe sodium intake and patient factors associated with sodium intake in community-dwelling persons with heart failure. ${ }^{9}$ The secondary purpose is to describe the overall dietary quality of persons with heart failure, based on additional nutrients documented as being beneficial to non-endstage heart failure and its underlying conditions. ${ }^{2-8,12}$ These include nutrients listed in the DASH recommendations: potassium, calcium, magnesium, fiber, saturated fat, protein and cholesterol, ${ }^{13}$ as well as omega-3 fish oil. ${ }^{10}$

\section{METHODS}

\section{Data Source and Population}

The NHANES is a series of repeated cross-sectional studies conducted by the Centers for Disease Control and Prevention to provide health information representative of the civilian, non-institutionalized, U.S. population. NHANES uses a multistage, stratified sampling design to ensure adequate represen- 
tation of the U.S. population. It consists of a questionnaire, physical examination and dietary assessment at a mobile examination center. ${ }^{14}$ Beginning in 1999, NHANES has collected data in contiguous 2-year cycles. The current analyses compiled data from the 1999-2000, 2001-2002, 2003-2004 and 2005-2006 cycles.

The population investigated in this study consisted of adults 50 years or older who reported ever being diagnosed having heart failure by a health care provider. Persons under age 50 were excluded because heart failure is uncommon among younger persons. ${ }^{15}$ Of the 8,825 adults age 50 years and over, $574(6.5 \%)$ reported having been diagnosed with heart failure.

\section{Dietary Assessments}

A single 24-hour dietary recall was administered to each participant by trained NHANES staff using the Food Intake Analysis System (FIAS). ${ }^{16}$ This consists of an automated computer-assisted interview which uses a five-step process with standardized wording to elicit recall of all foods and ingredients consumed. Prompts are provided to ensure complete descriptions of foods, preparation methods and portions. The database includes approximately 7,300 foods including brand names, ethnic foods and recipes, which allows calculation of nutrient information for 52 food components.

\section{Dietary Measures}

The primary nutrient of interest was sodium. This analysis used the heart failure-specific sodium intake recommendations from the American College of Cardiology/American Heart Association (ACC/AHA). ${ }^{9}$ Additional nutrients promoted by the DASH diet, which documented associations with impact on heart failure outcomes, were also assessed. ${ }^{2-8}$ The DASH diet promotes a high quality diet that includes consumption of fruits, vegetables, whole grains, low-fat and fat-free dairy products, lean meats, poultry and fish, and sparse intake of fats, oils, sweets, and added sugars. ${ }^{13,17}$ Nutrients included in the DASH diet are potassium, calcium, magnesium, fiber, saturated fat, protein and cholesterol. Omega-3 fish oil intake, using the AHA benchmark ${ }^{18}$, was also assessed because of evidence supporting its benefit among persons with heart failure. ${ }^{10,19}$

The ACC/AHA recommends that persons with heart failure follow a low sodium diet, defined as less than $2000 \mathrm{mg}$ per day. ${ }^{9}$ Other components of the DASH diet include potassium (. $\geq 4700 \mathrm{mg} /$ day), calcium ( $\geq 1240 \mathrm{mg} /$ day), magnesium ( $>500 \mathrm{mg} /$ day), fiber ( $>30 \mathrm{gm} /$ day) and cholesterol $(<150 \mathrm{mg}$ ) day). The DASH diet recommends that adults consume less than $6 \%$ of their energy intake from saturated fat and $\geq 18 \%$ of total energy intake from protein. These were calculated as number of saturated fat calories ( 9 calories/gm) or protein calories (4 calories/gm) consumed divided by total number of calories consumed. The AHA guidelines for persons with cardiovascular disease also recommend consumption one gram or more per day of eicosapentaenoic acid and docosahexaenoic acids, the omega 3 fatty acids in fish oils. ${ }^{18}$

\section{Co-variates}

Information was collected on socio-demographic characteristics including age, gender, race/ethnicity, education and income level. Body mass index (BMI) was calculated from measured height and weight $\left(\mathrm{kg} / \mathrm{m}^{2}\right)$ taken during the mobile examination. Current smoking status was created from first asking whether the participant had ever smoked 100 cigarettes in their lifetime, and if so, whether they currently smoked. NHANES survey year was also considered a potential covariate to account for potential differences across time periods. Common medical conditions known to contribute to heart failure development, coronary heart disease, hypertension, high cholesterol, and renal disease, were assessed by selfreport. Self-reported length of time since heart failure diagnosis was also assessed.

Diuretic use was classified into four categories according to potential biologic effect upon potassium: 1) thiazide, loop and carbonic acid inhibitor diuretics, which may cause hypokalemia; 2) potassium-sparing, aldosterone antagonist diuretics, and the combination of hydrochlorothiazide with triamterene, which may cause hyperkalemia; 3) other combinations with diuretics, which may be unpredictable with regard to effect upon potassium; and 4) no diuretic. ${ }^{20}$

\section{Statistical Analysis}

Frequency distributions were used to describe the population. Means (SE) for each dietary factor were computed. Percentages of persons with heart failure achieving each dietary goal were estimated. The associations of patient factors with sodium intake were analyzed using survey linear regression modeling. Each patient factor was first included in an unadjusted model. Variables significantly associated $(\mathrm{p} \leq 0.05)$ were entered into a multivariable model. Variables that remained statistically significant were retained in the final model. Analyses were carried out using Stata SE 10 (Stata Corp., College Station, Texas) and weighted using probability weights provided in the NHANES datasets as derived from estimates for the year 2000 population with respect to age, gender and race/ethnicity. ${ }^{14}$

\section{RESULTS}

\section{Study Population}

The study population consisted of an approximately equal proportion of men and women, with the majority (70\%) being greater than or equal to 65 years. The majority (80\%) were non-Hispanic white. Approximately one-third (34\%) had attained greater than a high school degree, and 53\% had been diagnosed with heart failure less than five years prior to the study interview. Table 1 .

\section{Sodium}

Table 2 presents a summary of sodium intake. Average intake was 2,728.5 ( $\mathrm{SE}=93.5) \mathrm{mg} /$ day. Only $33 \%$ achieved the AHA goal for persons with heart failure of less than $2,000 \mathrm{mg} /$ day. This intake was similar across diuretic categories: $2,756.0 \mathrm{mg} /$ day $(\mathrm{SE}=96.3)$ for those taking diuretic associated with potassium loss, 2,605.7 mg/day ( $\mathrm{SE}=202.8)$ for those taking a potassium-sparing diuretic, $2642.4 \mathrm{mg} /$ day $(\mathrm{SE}=149.5)$ for those taking a combination of diuretics, and $2,732.5 \mathrm{mg} /$ day $(\mathrm{SE}=90.7)$ for those not taking diuretics. Patient factors 
Table 1. Description of Persons Age 50 and Over with Selfreported Heart Failure, NHANES 1999-2000, 2001-2002, 2003-2004 and $2005-2006^{a}$

\begin{tabular}{|c|c|}
\hline Characteristic & Mean (SE) or \% \\
\hline Age, mean (SE) & $70.3(0.64)$ \\
\hline Female gender & $52.0 \%$ \\
\hline \multicolumn{2}{|l|}{ Race/ethnicity } \\
\hline Non-Hispanic white & $79.8 \%$ \\
\hline Non-Hispanic black & $11.4 \%$ \\
\hline Hispanic & $6.6 \%$ \\
\hline Other & $2.2 \%$ \\
\hline \multicolumn{2}{|l|}{ Education } \\
\hline$<$ High school degree & $41.8 \%$ \\
\hline High school degree & $24.3 \%$ \\
\hline > High school degree & $33.9 \%$ \\
\hline \multicolumn{2}{|l|}{ Annual income } \\
\hline$<\$ 25,000$ & $47.7 \%$ \\
\hline$\$ 25,000-\$ 55,000$ & $33.8 \%$ \\
\hline$>=\$ 55,000$ & $18.5 \%$ \\
\hline \multicolumn{2}{|l|}{ Body mass index } \\
\hline$<18.5 \mathrm{~kg} / \mathrm{m}^{2}$ & $0.6 \%$ \\
\hline $18.5-24.9 \mathrm{~kg} / \mathrm{m}^{2}$ & $20.0 \%$ \\
\hline $25.0-29.9 \mathrm{~kg} / \mathrm{m}^{2}$ & $36.3 \%$ \\
\hline$\geq 30.0 \mathrm{~kg} / \mathrm{m}^{2}$ & $43.1 \%$ \\
\hline Current smoker & $17.3 \%$ \\
\hline \multicolumn{2}{|l|}{ Number of years since told had heart failure } \\
\hline $0-2$ & $28.3 \%$ \\
\hline $3-5$ & $24.5 \%$ \\
\hline $6-10$ & $18.2 \%$ \\
\hline $11+$ & $28.9 \%$ \\
\hline Provider ever told had coronary heart disease & $66.1 \%$ \\
\hline Provider ever told had hypertension & $70.0 \%$ \\
\hline Provider ever told had high cholesterol & $61.4 \%$ \\
\hline Provider ever told had diabetes & $35.4 \%$ \\
\hline Provider ever told had kidney/renal disease & $5.6 \%$ \\
\hline \multicolumn{2}{|l|}{ Diuretic use } \\
\hline Taking diuretic that can cause potassium loss & $41.4 \%$ \\
\hline Taking potassium-sparing diuretic & $4.1 \%$ \\
\hline Taking combination of diuretics & $10.1 \%$ \\
\hline No diuretic & $44.0 \%$ \\
\hline Total calories per day, (SE) & $1714(38)$ \\
\hline
\end{tabular}

${ }^{a}$ Data weighted to represent the total U.S. population with heart failure

associated with sodium intake in the linear regression model are presented in Table 2. Female gender, higher education, higher income and self-reported diagnosis of hypertension were associated with lower sodium intake.

\section{Additional Nutrients}

Table 3 presents mean (SE) nutrient intake and the percentage of persons that met each additional dietary goal. Overall, $3.1 \%$ reported intake of $4,700 \mathrm{mg} /$ day or more of potassium. There were non-significant differences in potassium intake according to use of diuretics associated with potassium loss (mean=2,315.8, $\mathrm{SE}=72.4$ ), use of potassium-sparing diuretics (mean=2,439.3, $\mathrm{SE}=282.7$ ), combination of diuretics (mean= $2,407.5, \mathrm{SE}=112.5)$ or not taking any diuretic (mean=2,396.7, $\mathrm{SE}=73.2$ ).

The percentages of participants that met the intake goals for calcium and magnesium were $11.6 \%$ and $2.5 \%$, respectively. Less than four percent $(3.8 \%)$ met the fiber intake goal. The adherence rate for saturated fat was $13.6 \%$ and for fish oil was $2.3 \%$. Approximately one-quarter $(26.7 \%)$ met the protein goal, while $40.4 \%$ met the cholesterol goal.
Table 2. Description of Sodium Intake and Factors Associated with Increased Sodium Intake Among Persons Age 50+ with Heart Failure in NHANES 1999-2000, 2001-2002, 2003-2004, 2005-2006 ${ }^{\circ}$

\begin{tabular}{|c|c|c|}
\hline \multicolumn{3}{|l|}{ Daily sodium intake } \\
\hline Mean daily intake (SE) & \multicolumn{2}{|l|}{$2,728.5(93.5)$} \\
\hline Sodium category & \multicolumn{2}{|l|}{$\%$ of population } \\
\hline$<1500 \mathrm{mg}$ & \multicolumn{2}{|l|}{$18.7 \%$} \\
\hline $1500-1999 \mathrm{mg}$ & \multicolumn{2}{|l|}{$15.3 \%$} \\
\hline $2000-2499 \mathrm{mg}$ & \multicolumn{2}{|l|}{$18.4 \%$} \\
\hline $2500-2999 \mathrm{mg}$ & \multicolumn{2}{|l|}{$15.6 \%$} \\
\hline $3000-3999 \mathrm{mg}$ & \multicolumn{2}{|l|}{$16.6 \%$} \\
\hline $4000+\mathrm{mg}$ & \multicolumn{2}{|l|}{$15.4 \%$} \\
\hline \multicolumn{3}{|c|}{$\begin{array}{l}\text { Multivariable linear regression model of factors associated with } \\
\text { increasing daily sodium intake }\end{array}$} \\
\hline Characteristic & $\beta$ (unit $=\mathrm{mg} /$ day) (SE) & $\mathrm{P}$-value \\
\hline Female gender & $-577.1(119.2)$ & $<0.0001$ \\
\hline \multicolumn{3}{|l|}{ Education } \\
\hline$<$ High school degree & Referent & \\
\hline High school degree & $-187.8(134.5)$ & 0.16 \\
\hline > High school degree & $-309.4(159.3)$ & 0.05 \\
\hline \multicolumn{3}{|l|}{ Annual income } \\
\hline$<\$ 25,000$ & Referent & \\
\hline$\$ 25,000-\$ 55,000$ & $-241.5(134.8)$ & 0.07 \\
\hline$>=\$ 55,000$ & $-839.7(175.6)$ & $<0.0001$ \\
\hline $\begin{array}{l}\text { Provider ever told had } \\
\text { high blood pressure }\end{array}$ & $-196.2(121.2)$ & 0.05 \\
\hline
\end{tabular}

${ }^{a}$ Data weighted to represent the total U.S. population with heart failure

\section{DISCUSSION}

High quality dietary patterns, characterized by adherence to sodium restriction and nutrients promoted in the DASH eating plan and consumption of omega-3 fish oils, have been associated with decreased risk and improved management of cardiovascular disease risk factors and conditions, including heart failure. ${ }^{2-8,11,12}$ This analysis demonstrates that the dietary quality of U.S. adults living in the community with heart failure is poor. It is likely the poor dietary patterns observed in the population began many years before diagnosis of heart failure, and in fact contributed to the development of heart failure. ${ }^{21}$ Indeed, studies examining adherence to DASH diet recommendations in populations at increased risk for developing heart failure (i.e. hypertension and atherosclerosis) have observed poor dietary quality. ${ }^{22,23}$ This study extends previous results by documenting that among persons with a severe chronic illness, namely heart failure, poor dietary habits

Table 3. Mean (SE) Dietary Intake of Additional Nutrients and Percentage Meeting Dietary Goals of Persons Age 50 and Over with Self-reported Heart Failure, NHANES 1999-2000, 2001-2002, 2003-2004 and 2005-2006

\begin{tabular}{llcc}
\hline \hline Dietary nutrient & Daily goal & Mean (SE) & $\begin{array}{l}\% \text { Meeting } \\
\text { goal }\end{array}$ \\
\hline Potassium & $\geq 4700 \mathrm{mg} /$ day & $2373,2(47.3)$ & $3.1 \%$ \\
Calcium & $\geq 1240 \mathrm{mg} /$ day & $705.6(21.0)$ & $11.6 \%$ \\
Magnesium & $\geq 500 \mathrm{mg} /$ day & $236.9(4.8)$ & $2.5 \%$ \\
Fiber & $\geq 30 \mathrm{~g} / \mathrm{d}$ & $13.6(0.4)$ & $3.8 \%$ \\
Fish oil \pm & $\geq 1 \mathrm{gm} /$ day & $0.11(0.01)$ & $2.3 \%$ \\
Saturated fat & $\leq 6 \%$ of total energy & $11.0(0.18)$ & $13.6 \%$ \\
Protein & $\geq 18 \%$ of total energy & $15.8(0.22)$ & $26.9 \%$ \\
Cholesterol & $\leq 150 \mathrm{mg} / \mathrm{d}$ & $262.4(11.7)$ & $40.4 \%$
\end{tabular}

${ }^{a}$ Data weighted to represent the total U.S. population with heart failure \pm Not included in DASH diet 
persist. Our findings reinforce the need for primary prevention efforts aimed at improving dietary quality for cardiovascular disease risk reduction.

Sodium restriction is the mainstay of heart failure dietary recommendations, ${ }^{9}$ yet adherence to established guidelines was poor. Because of impaired neurohormonal regulation of sodium excretion, patients with heart failure must attain a dietary sodium intake that is less than that of the general population. ${ }^{5}$ The average daily sodium intake among persons with heart failure in this analysis was $2,728.5 \mathrm{mg} /$ day, with $34 \%$ achieving the $2,000 \mathrm{mg} /$ day benchmark. The average daily sodium intake in the adult 1999-2006 NHANES sample age 50 and over without heart failure was $3,073.1 \mathrm{mg} /$ day (data not shown). Results suggest that even though sodium intake among persons with heart failure remains high, there has been some success in decreasing sodium intake among persons with heart failure at the population-level.

Sodium restriction is challenging because it limits restaurant fare and convenience foods. Further, it requires careful label reading and food preparation. This analysis found that persons with hypertension consumed approximately $200 \mathrm{mg} /$ day less than those without it, suggesting modest success of clinical and public health efforts to decrease sodium consumption in this atrisk group. Men and persons of lower education and income level had high sodium intake whereas persons of higher educational attainment and persons with higher income levels had lower sodium intake in this analysis. Educational attainment may be associated with better ability to read and understand food labels, while persons with higher income are allowed more choices about the foods that they purchase and consume. Targeted clinical efforts may be required to address differences in sodium intake according to socio-economic status indicators (education and income) and gender. However, given the widespread use of sodium in the food supply, any meaningful strategy to reduce sodium intake must rely upon a public and professional demand that manufacturers and restaurants reduce the amount of salt added during food preparation.

Potassium intake is of concern in heart failure. Low serum potassium has been shown to increase disease progression and increase all-cause mortality in persons with heart failure. ${ }^{24}$ Heart failure and its associated medications may alter serum potassium through changes in renal clearance and in the amounts and impact of aldosterone, renin-angiotensin and catecholamine secretions. Potassium deficiency in heart failure may result from a poor diet, anorexia, hyperaldosteronism, or through the use of loop or thiazide diuretics. ${ }^{25}$ Thus, eating high-potassium foods is recommended for many patients with heart failure. However, due to the risk of hyperkalemia in patients on potassium-sparing diuretics and those with renal failure, dietary potassium intake needs vary considerably, making it difficult to establish a universal goal. The results of this study demonstrate that overall, dietary potassium intake in persons with heart failure is low and that intake does not differ according to diuretic use. Because potassium has a narrow range and delicate physiological balance, potassium levels should be closely monitored for physicians to make individualized recommendations.

Calcium intake in this study was also inadequate. Calcium is essential for many regulatory functions affecting heart health, including coagulation/hemostasis, endocrine, exocrine, and neuroendocrine cell secretions, and actions of cardiac and skeletal muscles. ${ }^{26}$ Dietary calcium is implicated for its beneficial role in heart failure, as part of electrolyte balance to help maintain optimal heart contractility and rhythm, and blood pressure measures. ${ }^{27}$ Calcium appears to have a stabilizing effect on muscle contraction to prevent vasoconstriction in "calcium sensitive" hypertensives. ${ }^{27}$ Calcium may also directly inhibit renal sodium reabsorption, and/ or lower the activity of the renin-angiotensin system to benefit hypertension. ${ }^{28}$ Unfortunately, loop diuretics may cause hypocalcemia. ${ }^{29}$ Thus, insufficient intake of calcium, flagged by the 2005 Dietary Guidelines for Americans (DGA 2005) as a nutrient of concern for most Americans, ${ }^{30}$ is of particular concern among persons with heart failure.

The DGA 2005 also named magnesium a nutrient of concern. ${ }^{30}$ Magnesium primarily affects cardiovascular, neuromuscular, and renal tissues. Magnesium intake may temper the increased arrhythmic risk and vasoconstriction experienced by many heart failure patients. ${ }^{6,31}$ Magnesium deficiency can lead to increased sodium retention, decreased potassium uptake, and affect essential intracellular calcium concentrations, all of which further complicate the management of heart failure. Persons taking loop and/or thiazide diuretic treatments for heart failure may need an even higher magnesium intake to address increased urinary excretion of magnesium caused by these medications.

Fiber intake was also inadequate. Fiber can impact inflammation, weight, blood pressure, glycemic control, and other cardiovascular disease biomarkers. ${ }^{32}$ Because fiber can be a dietary addition, rather than a restriction, adherence to this goal may be enhanced if routinely recommended.

Omega-3 fatty acids, specifically eicosapentaenoic acid (EPA) and docosahexaenoic acid (DHA) derived from fish, have been shown to suppress inflammatory markers and increase adiponectin for patients with heart failure. ${ }^{18,19}$ Since fish is a source of protein, potassium, and omega-3 fatty acids, following the AHA recommendation to eat at least two fatty fish meals per week may increase adherence to several nutrient goals. Only a small percentage of the NHANES study sample met intake goals for saturated fat. A cornerstone of dietary recommendations for persons with cardiovascular disease, excess intake of saturated fat has been directly correlated with increased LDL cholesterol, ${ }^{33}$ and increased levels of proinflammatory cytokines. ${ }^{7,34-37}$ A low saturated fat diet is particularly important in heart failure, to address a markedly increased risk for cardiovascular disease complications.

Protein energy malnutrition is generally not of concern in the U.S. population, ${ }^{38}$ yet $27 \%$ of this population did not achieve the DASH benchmark of $18 \%$ of total calories. Because common protein sources include foods that are also high in saturated fat (e.g. red meats) and sodium (e.g. processed meats), public health messages should reinforce the importance of obtaining protein from food sources that are otherwise beneficial.

Cholesterol presents an interesting case among dietary recommendations. It has not been definitively linked, as has saturated fat, with a detrimental lipidemic effect, because it tends to raise both LDL and HDL cholesterol. ${ }^{39}$ Studies suggest that the association between dietary cholesterol and occurrence of heart disease is small; ${ }^{40,41}$ and of the nutrients listed above, cholesterol is probably the least important. However, given that patients appear to be at least somewhat (40\%) adherent to this recommendation, it may present an opportunity to impact other beneficial nutrients since lowering dietary cholesterol might reduce the intake of foods that also contain saturated fat. 
This study has several limitations. The sample size $(\mathrm{n}=574)$ was relatively small. Self-reported diagnosis may under-estimate the true proportion of the population that has chronic heart failure. ${ }^{42}$ It is possible that the dietary status of persons who do not report having heart failure, either by choice or because they do not know or understand that they have the disease, is even poorer than what was observed. This study is cross-sectional, so change in dietary habits as a result of diagnosis is unknown. Dietary recall data have limitations. People tend to under-report consumption of calories and certain nutrients, including saturated fat and sodium, which would point to even poorer intake. ${ }^{43,44}$ Diet was assessed by a single 24 -hour dietary recall. Though a single 24-hour dietary recall is not suitable for assessing individual intake, it is appropriate for determining group means. ${ }^{45}$ Doses and timing of dose changes were not available, nor were blood levels of potassium, sodium or other nutrients, so diuretic influence upon physiological status could not be determined. Data were not available for supplemental potassium and calcium consumption, over-the-counter calcium supplements or salt-substitutes with potassium. Thus, the results presented likely under-estimate total consumption and represent intake through diet. Data were also not available for heart failure symptoms. The fact that almost half of patients were not on a diuretic implies that they did not have symptoms. However, sodium consumption did not differ by diuretic status, suggesting that intake did not differ by symptom status.

\section{CONCLUSIONS}

The results of this study suggest that the dietary quality of persons with heart failure is poor, which has strong potential for detrimental effect on disease progression and underlying conditions. Numerous approaches will likely be required to address this issue. Patients need clear and simple dietary prescriptions for specific foods and food groups from health care providers. Moreover, effective, large-scale intervention strategies are needed $^{11}$ because making dietary changes, even in the presence of life threatening illness, is extremely difficult. ${ }^{46}$ Such strategies should address both primary and secondary prevention. Results also support the need for public health approaches. In particular, since non-discretionary sodium added to foods is so prevalent, increased public and political pressure is needed to make changes to the food supply to improve the diet of persons with heart failure and the population in general.

Acknowledgement: This work was presented at the Society of Behavioral Medicine 2009 annual conference.

Conflict of Interest: None disclosed.

Corresponding Author: Stephenie C. Lemon, $\mathrm{PhD}$; Division of Preventive and Behavioral Medicine, S7-745, University of Massachusetts Medical School, 55 Lake Avenue North, Worcester, MA 01655, USA (e-mail: Stephenie.Lemon@umassmed.edu).

\section{REFERENCES}

1. Rosamond $\mathbf{w}$, Flegal $\mathbf{K}$, Furie $\mathbf{K}$, et al. Heart disease and stroke statistics-2008 update: a report from the American Heart Association Statistics Committee and Stroke Statistics Subcommittee. Circulation 2008;117(4):e25-146.
2. Alsafwah S, Laguardia SP, Arroyo M, et al. Congestive heart failure is a systemic illness: a role for minerals and micronutrients. Clin Med Res. 2007;5(4):238-43.

3. Newman KP, Bhattacharya SK, Munir A, Davis RC, Soberman JE, Ramanathan KB. Macro- and micronutrients in patients with congestive heart failure, particularly African-Americans. Vasc Health Risk Manag. 2007;3(5):743-7.

4. Bhattacharya SK, Ahokas RA, Carbone LD, et al. Macro- and micronutrients in African-Americans with heart failure. Heart Fail Rev. 2006;11(1):45-55.

5. Selektor Y, Weber KT. The salt-avid state of congestive heart failure revisited. Am J Med Sci. 2008;335(3):209-18.

6. Witte KK, Clark AL. Micronutrients and their supplementation in chronic cardiac failure. An update beyond theoretical perspectives. Heart Fail Rev. 2006;11(1):65-74

7. Lennie TA, Chung ML, Habash DL, Moser DK. Dietary fat intake and proinflammatory cytokine levels in patients with heart failure. J Card Fail. 2005; 11(8):613-8.

8. Morgan DR, Dixon LJ, Hanratty CG, et al. Effects of dietary omega-3 fatty acid supplementation on endothelium-dependent vasodilation in patients with chronic heart failure. Am J Cardiol. 2006;97(4):547-51.

9. Hunt SA. ACC/AHA 2005 guideline update for the diagnosis and management of chronic heart failure in the adult: a report of the American College of Cardiology/American Heart Association Task Force on Practice Guidelines (Writing Committee to Update the 2001 Guidelines for the Evaluation and Management of Heart Failure). J Am Coll Cardiol. 2005;46(6):e1-82.

10. Duda MK, O'Shea KM, Stanley WC. \{omega\}-3 polyunsaturated fatty acid supplementation for the treatment of heart failure: mechanisms and clinical potential. Cardiovasc Res. 2009; Epub ahead of print.

11. Ershow AG, Costello RB. Dietary guidance in heart failure: a perspective on needs for prevention and management. Heart Fail Rev. 2006;11 (1):7-12.

12. Redfield MM, Jacobsen SJ, Burnett JC Jr, Mahoney DW, Bailey KR, Rodeheffer RJ. Burden of systolic and diastolic ventricular dysfunction in the community: appreciating the scope of the heart failure epidemic. JAMA. 2003;289(2):194-202.

13. Svetkey LP, Simons-Morton D, Vollmer WM, et al. Effects of dietary patterns on blood pressure: subgroup analysis of the Dietary Approaches to Stop Hypertension (DASH) randomized clinical trial. Arch Intern Med. 1999;159(3):285-93.

14. Centers for Disease Control and Prevention (CDC). National Center for Health Statistics (NCHS). Health and Nutrition Examination Survey Data. 1999-2006. Available at: http://www.cdc.gov/nchs/nhanes/ nhanes_questionnaires.htm. Accessed 9/3/2009.

15. Kannel WB. Incidence and epidemiology of heart failure. Heart Fail Rev. 2000;5(2): 167-73.

16. University of Texas Health Science Center at Houston. Food Intake Analysis System. Available at: http://www.sph.uth.tmc.edu/DellHealthyLiving/ default.aspx?id=4008 Accessed 9/3/2009.

17. Appel LJ, Champagne CM, Harsha DW, et al. Effects of comprehensive lifestyle modification on blood pressure control: main results of the PREMIER clinical trial. JAMA. 2003;289(16):2083-93.

18. Kris-Etherton PM, Harris WS, Appel LJ. Fish consumption, fish oil, omega-3 fatty acids, and cardiovascular disease. Circulation. 2002;106 (21):2747-57.

19. Duda MK, O'Shea KM, Tintinu A, et al. Fish oil, but not flaxseed oil, decreases inflammation and prevents pressure overload-induced cardiac dysfunction. Cardiovasc Res. 2009;81(2):319-27.

20. American Society of Health-System Pharmacists. AHFS Drug Information 2009. Bethesda, MD: ASHP; 2009.

21. Levitan EB, Wolk A, Mittleman MA. Consistency with the DASH diet and incidence of heart failure. Arch Intern Med. 2009;169(9):851-7.

22. Mellen PB, Gao SK, Vitolins MZ, Goff DC Jr. Deteriorating dietary habits among adults with hypertension: DASH dietary accordance, NHANES 1988-1994 and 1999-2004. Arch Intern Med. 2008;168 (3):308-14.

23. Gao SK, Fitzpatrick AL, Psaty B, et al. Suboptimal nutritional intake for hypertension control in 4 ethnic groups. Arch Intern Med. 2009; 169 (7):702-7.

24. Ahmed A, Zannad F, Love TE, et al. A propensity-matched study of the association of low serum potassium levels and mortality in chronic heart failure. Eur Heart J. 2007;28(11):1334-43.

25. Parker B, Johnson PJ. Heart Failure. In: Di Piro JT, TAlbert RL, Yee GC, eds. Pharmacotherapy: A Pathophysiologic Approach. McGraw-Hill; 2005, pp. 219-260. 
26. Hunt SM, Groff JL. Advanced Nutrition and Human Metabolism. St. Paul, MN: West Publishing Company; 1990.

27. Reusser ME, McCarron DA. Reducing hypertensive cardiovascular disease risk of African Americans with diet: focus on the facts. J Nutr. 2006;136(4):1099-102.

28. Zemel MB, Richards J, Milstead A, Campbell P. Effects of calcium and dairy on body composition and weight loss in African-American adults. Obesity Res. 2005;13(7):1218-25.

29. Dunn SP, Bleske B, Dorsch M, Macaulay T, Van Tassell B, Vardeny O. Nutrition and heart failure: impact of drug therapies and management strategies. Nutr Clin Pract. 2009;24(1):60-75.

30. US Department of Health and Human Services, US Department of Agriculture. Dietary guidelines for Americans 2005, 6th edition. Washington, D.C.: US Government Printing Office, 2005.

31. Bourdel-Marchasson I, Emeriau JP. Nutritional strategy in the management of heart failure in adults. Am J Cardiovasc Drugs. 2001;1(5):363-73.

32. Anderson JW, Smith BM, Gustafson NJ. Health benefits and practical aspects of high-fiber diets. Am J Clin Nutr. 1994;59(5 Suppl):1242S-7S.

33. Lichtenstein AH, Appel LJ, Brands M, et al. Diet and lifestyle recommendations revision 2006: a scientific statement from the American Heart Association Nutrition Committee. Circulation. 2006;114(1):82-96.

34. Ma Y, Griffith JA, Chasan-Taber L, et al. Association between dietary fiber and serum C-reactive protein. Am J Clin Nutr. 2006;83(4):760-6.

35. Galisteo M, Duarte J, Zarzuelo A. Effects of dietary fibers on disturbances clustered in the metabolic syndrome. J Nutr Biochem. 2008;19(2):71-84.

36. Ma Y, Hebert JR, Li W, et al. Association between dietary fiber and markers of systemic inflammation in the Women's Health Initiative Observational Study. Nutrition. 2008;24(10):941-9.

37. Van Horn L. Fiber, lipids, and coronary heart disease. A statement for healthcare professionals from the Nutrition Committee, American Heart Association. Circulation. 1997;95(12):2701-4.
38. Rabinowitz SS, Gehri M, Di Paolo ER, Wetterer NM. Marasmus. [website] 2009 May 20,2009 Available at: http://www.emedicine.com/ ped/TOPIC164.HTM\#section Introduction. Accessed 9/2/2009.

39. Herron KL, Lofgren IE, Sharman M, Volek JS, Fernandez ML. High intake of cholesterol results in less atherogenic low-density lipoprotein particles in men and women independent of response classification. Metabolism. 2004;53(6):823-30.

40. Kratz M. Dietary cholesterol, atherosclerosis and coronary heart disease. Handb Exp Pharmacol. 2005;170:195-213.

41. Vestfold Heartcare Study Group. Influence on lifestyle measures and five-year coronary risk by a comprehensive lifestyle intervention programme in patients with coronary heart disease. Eur J Cardiovasc Prev Rehabil. 2003;10(6):429-37.

42. Okura Y, Urban LH, Mahoney DW, Jacobsen SJ, Rodeheffer RJ. Agreement between self-report questionnaires and medical record data was substantial for diabetes, hypertension, myocardial infarction and stroke but not for heart failure. J Clin Epidemiol. 2004;57(10):1096103

43. Subar AF, Kipnis V, Troiano RP, et al. Using intake biomarkers to evaluate the extent of dietary misreporting in a large sample of adults: the OPEN study. Am J Epidemiol. 2003;158(1):1-13.

44. Kipnis V, Subar AF, Midthune D, et al. Structure of dietary measurement error: results of the OPEN biomarker study. Am J Epidemiol 2003;158(1): 14-21. discussion 22-6.

45. Beaton GH, Milner J, McGuire V, Feather TE, Little JA. Source of variance in 24-hour dietary recall data: implications for nutrition study design and interpretation. Carbohydrate sources, vitamins, and minerals. Am J Clin Nutr. 1983;37(6):986-95.

46. Ma Y, Li W, Olendzki BC, et al. Dietary quality 1 year after diagnosis of coronary heart disease. J Am Diet Assoc. 2008;108(2):240-6. discussion $246-7$ 